\title{
Applying design patterns for web-based derivatives pricing
}

\author{
V. Papakostas, P. Xidonas, D. Askounis \& J. Psarras \\ School of Electrical and Computer Engineering, \\ National Technical University of Athens, Greece
}

\begin{abstract}
Derivatives pricing models have been widely applied in the financial industry for building software systems for pricing derivative instruments. However, most of the research work on financial derivatives is concentrated on computational models and formulas. There is little guidance for quantitative developers on how to apply these models successfully in order to build robust, efficient and extensible software applications. The present paper proposes an innovative design of a web-based application for real-time financial derivatives pricing, which is entirely based on design patterns, both generic and web-based application specific. Presentation tier, business tier and integration tier patterns are applied. Financial derivatives, underlying instruments and portfolios are modelled. Some of the principal models for evaluating derivatives (Black-Scholes, binomial trees, Monte Carlo simulation) are incorporated. Arbitrage opportunities and portfolio rebalancing requirements are detected in real time with the help of a notification mechanism. The novelty in this paper is that the latest trends in software engineering, such as the development of webbased applications, the adoption of multi-tiered architectures and the use of design patterns, are combined with financial engineering concepts to produce design elements for software applications for derivatives pricing. Although our design best applies to the popular J2EE technology, its flexibility allows many of the principles presented to be adopted by web-based applications implemented with alternative technologies.

Keywords: financial applications, financial derivatives, pricing models, design patterns, J2EE patterns, web-based applications, multi-tiered architectures.
\end{abstract}




\section{Introduction}

Financial derivatives have become extremely popular among investors for hedging and speculating. Their growing use has triggered an increased interest in financial engineering and the emergence of several computational models for evaluating them and determining their characteristics.

Numerous software systems and applications have been developed for implementing such models. Some are in-house applications for large financial institutions and investment banks while others are available as software products. Despite the plethora of computational models that are present in the relevant literature, the existence of books and publications on the design and construction of software systems for implementing them is limited. Even these are usually constrained to conventional object-oriented design, circumstantial use of design patterns and traditional programming languages like $\mathrm{C}++$ or Visual Basic.

The objective of the present paper is to propose an innovative design of a web-based application for real-time financial derivatives and portfolios pricing. The modelled application quotes derivatives and underlying assets prices from market data feeds and applies pricing models for computing derivatives and portfolios theoretical values and characteristics. In addition to rendering pricing information on web pages, it can send notifications (e.g. emails) when prices or attributes of derivatives or portfolios satisfy certain conditions (e.g. permit arbitrage or require portfolio rebalancing).

Design patterns play central role in our design, upon which it is almost entirely based. Both generic [5] and web-based applications specific patterns (J2EE patterns [1]) are applied. The proposed design aims to facilitate the introduction of new derivative instruments, additional valuation models and alternative market data feeds to the system on subsequent phases after its initial release.

\section{Background work}

Joshi [7] and Duffy [3] apply the concepts of object-oriented programming and adopt design patterns for evaluating financial derivatives. London [9] assembles a number of pricing models implemented in the $\mathrm{C}++$ programming language. Zhang and Sternbach [12] model financial derivatives using design patterns. van der Meij et al [11] describe the adoption of design patterns in a derivatives pricing application. Marsura [10] presents a complete application for evaluating derivatives and portfolios using objects and design patterns. Eggenschwiler and Birrer [2], and Gamma and Eggenschwiler [4] describe the use of objects and frameworks in financial engineering. Koulisianis et al [8] present a web-based application for derivatives pricing implemented with the PHP technology, using the Problem Solving Environment methodology. 


\section{Derivatives pricing models}

It is possible to estimate the value that a financial derivative contract should theoretically have from the underlying asset price and the contract characteristics. If the difference between the market price and the theoretical value of the contract is significant, an investor can achieve guaranteed profit (arbitrage). For this reason, derivatives pricing has become the field of extensive study for the past three decades.

A number of pricing models (analytical and numerical methods) have emerged and applied for derivatives pricing [6]. Black-Scholes equation provides analytical formulas for calculating theoretical prices of European call and put options on non-dividend paying stocks. Binomial trees are particularly useful in cases that an option holder has the potential for early exercise. Monte Carlo simulation is primarily applied when the derivative price depends on the history of the underlying asset price or on multiple stochastic variables.

\section{Multi-tiered architecture}

The present paper proposes the design of an application for derivatives pricing that is web-based. The use of the internet introduces certain complexity into our model. A multi-tiered architecture has been adopted for our design. Each tier in a multi-tiered architecture is responsible for a subset of the system functionality [1]. It is logically separated from its adjacent tiers and loosely connected to them. It is important to emphasise that a multi-tiered architecture is logical and not physical. This means that multiple tiers may be deployed on a single machine or a single tier may be deployed on multiple machines, especially if it contains CPU intensive components.

\section{Design patterns}

\subsection{Presentation tier}

\subsubsection{Front Controller}

The Font Controller pattern forms the initial point of communication for handling user requests, aiming to reduce the administration and deployment tasks for the application [1]. One Front Controller is used for all user requests. It is incarnated by the FrontController class, which is a servlet.

\subsubsection{Context Object}

The Context Object pattern encompasses state in a protocol independent way, in order to be utilized by different parts of the application [1]. One Context Object is used for each type of user request. Requests for futures pricing are modelled by the FuturePricingRequestContext class, requests for options pricing by the OptionPricingRequestContext class, etc. The Factory pattern is applied for their creation. 


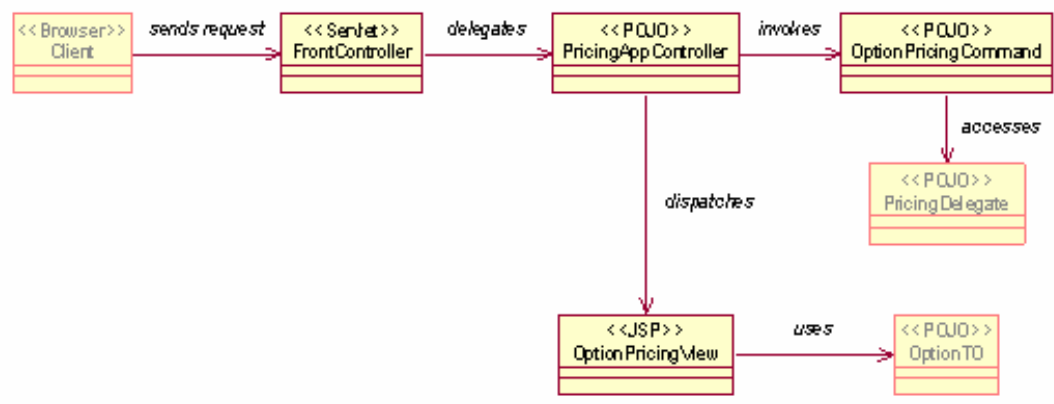

Figure 1: Presentation tier design patterns.

\subsubsection{Application Controller}

The Application Controller pattern centralizes the invocation of actions for handling requests (action management) and the dispatch of response data to the proper view (view management) [1]. Our design suggests the use of the ApplicationController interface for modelling Application Controller functionality. The PricingAppController class, which implements this interface, coordinates Commands and Views related to pricing requests. The ManagementAppController class does the same for requests related to instrument management, such as adding a new financial instrument to the application. The Factory pattern is again applied for their creation.

\subsubsection{View Helper}

The View Helper pattern uses views to encapsulate the code that formats responses to user requests and helpers to encapsulate the logic that views require in order to obtain response data [1]. In our design, Views are incarnated by a number of JSP pages. PortfolioDetailsView displays information related to portfolios definition, OptionPricingView displays the results of an option pricing request, etc. Business Delegates are used as Helpers.

\subsubsection{Command}

The Command pattern encapsulates the action required as the result of a request through the invocation of the corresponding functionality [5]. One Command is used for each type of user request. Requests for futures definition invoke class FutureDefinitionCommand, requests for portfolio pricing class PortfolioPricingCommand, etc. As a result, there is one-to-one correspondence among Context Objects and Commands. The Factory pattern is applied for their creation.

\subsubsection{Factory}

The Factory pattern is responsible for the creation of objects that implement an interface or extend an abstract class. In our design, a number of classes adopt this 
pattern, such as RequestContextFactory for the creation of Context Objects, ApplicationControllerFactory for the creation of Application Controllers, CommandFactory for the creation of Commands, etc. Factories can be configured declaratively through the use of XML files.

\subsubsection{Singleton}

The Singleton pattern defines classes that are allowed to have only one instance per application [5]. Each class that adopts the Factory pattern in our design adopts the Singleton pattern in addition.

\subsection{Business tier}

\subsubsection{Business Delegate}

The Business Delegate pattern encapsulates access to business services, aiming to reduce interconnection between components of the presentation and business tiers [1]. In our design, one Business Delegate is defined for each Session Façade. The PricingDelegate class provides centralised access to the PricingFacade class, the ManagementDelegate class to the ManagementFacade class and the NotificationDelegate class to the NotificationFacade class.

\subsubsection{Service Locator}

The Service Locator pattern centralises the lookup of services and components [1]. One Service Locator, which is incarnated by the ServiceLocator class, is used. It also adopts the Singleton pattern.

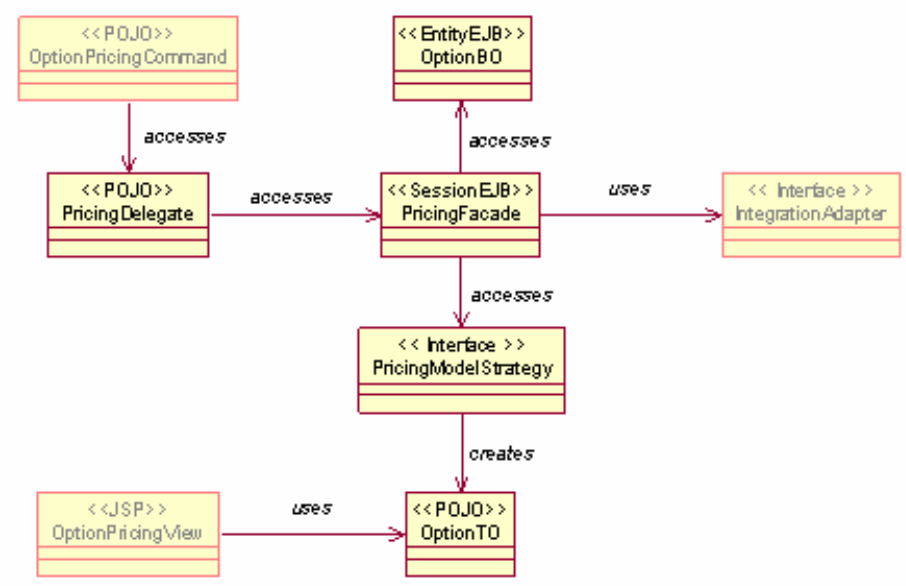

Figure 2: Business tier design patterns.

\subsubsection{Session Façade}

The Session Façade pattern encapsulates components of the business tier and exposes coarse-grained services to remote clients, aiming to reduce the number 
of remote method invocations among components of the presentation and business tiers [1]. Services related to derivatives and portfolios pricing are aggregated to the PricingFacade class. Services related to derivatives and portfolios management are encapsulated in the ManagementFacade class. Services for the notification mechanism are accumulated in the NotificationFacade class.

\subsubsection{Application Service}

The Application Service pattern underlies components that encapsulate business logic, aiming to leverage related services and objects (Business Objects) [1]. Our design adopts the layer strategy in regard to the use of Application Services. The PricingAppService and NotificationAppService classes, which reside on the higher layer, expose pricing and notification services respectively. They require pricing modelling related functionality, which is provided by the PricingModelStrategy interface, which resides on the lower layer, along with the BlackScholesAppService, BinomialTreeAppService and MonteCarloAppService classes that implement it. Financial instruments volatility is calculated on a daily basis by the VolatilityAppService class.

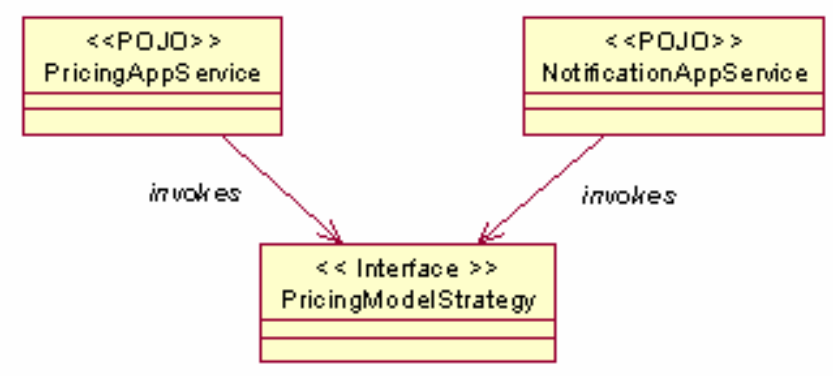

Figure 3: Application Service layering.

\subsubsection{Business Object}

The Business Object pattern encapsulates and administers business data, behaviour and persistence, aiming at the creation of objects with high cohesion [1]. Our design contains a hierarchy of Business Objects that correspond to portfolios and financial instruments. They consist of abstract classes FinancialInstrumentBO, Derivative $B O$ and concrete classes PortfolioBO, StockBO, IndexBO, CurrencyBO, FutureBO, OptionBO, EuropeanOptionBO, and AmericanOptionBO. This way, new derivative types can be added with minor modifications.

\subsubsection{Composite Entity}

The Composite Entity pattern aggregates a set of related Business Objects into one coarse-grained entity bean, allowing for the implementation of parent objects that manage dependent objects [1]. In our design, the PortfolioBO class, which 
represents a portfolio, is defined as parent object and the PortfolioInstrument class, which represents a financial instrument that is member of a portfolio, as dependent object. Although a PortfolioInstrument object is linked to a FinancialInstrument $B O$ object, it is a separate object. It holds information such as quantity and (call/put) position of a specific instrument in a portfolio.

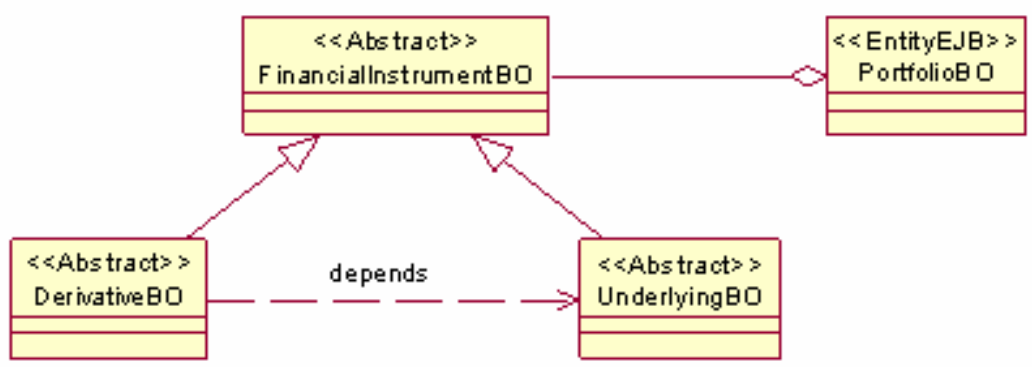

Figure 4: Business Objects for financial instruments.

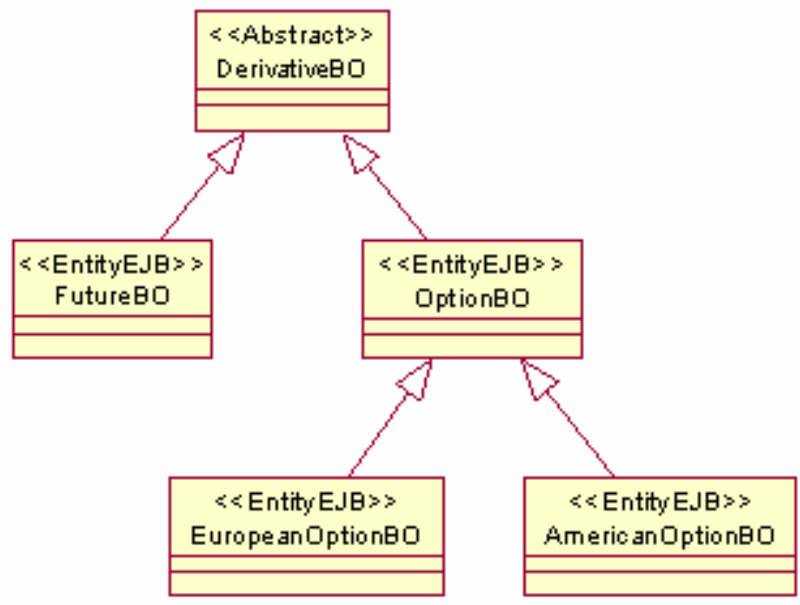

Figure 5: Hierarchy of Business Objects for derivatives.

\subsubsection{Transfer Object}

The Transfer Object (or Data Transfer Object) pattern carries multiple data across application tiers [1]. Our design adopts the multiple transfer objects strategy in regard to the use of Transfer Objects. One Transfer Object is defined for each Business Object. This leads to a hierarchy of Transfer Objects that correspond to financial instruments and portfolios. They consist of classes 
FinancialInstrumentTO, DerivativeTO, PortfolioTO, StockTO, IndexTO, CurrencyTO, FutureTO, OptionTO, EuropeanOptionTO, and AmericanOptionTO.

\subsubsection{Strategy}

The Strategy pattern encapsulates a family of algorithms and makes them interchangeable [5]. Considering our design, such algorithms are the pricing models for derivatives. The PricingModelStrategy interface adopts this pattern. It is implemented by the BlackScholesAppService, BinomialTreeAppService and MonteCarloAppService classes, which contain the algorithms for the Black-Scholes, binomial trees and Monte Carlo models respectively. This way, new pricing models can be introduced with minor modifications.

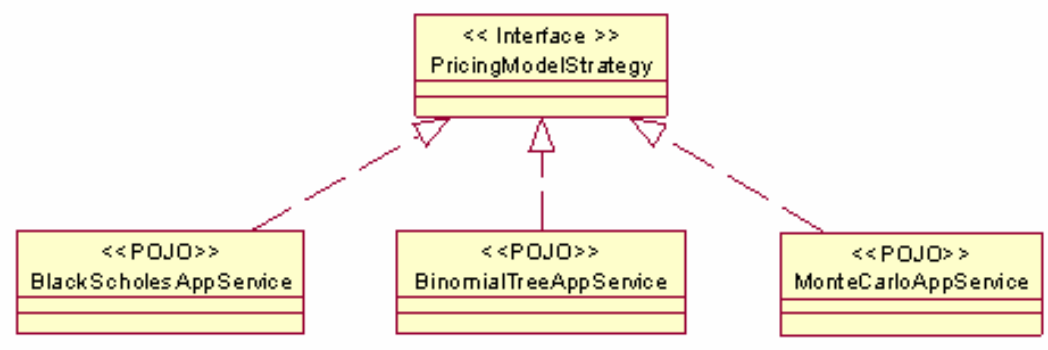

Figure 6: Strategy.

\subsubsection{Observer}

The Observer pattern defines an one-to-many correspondence between an observable object (Observable or Publisher) and one or more observer objects (Observers or Subscribers). When the observable object changes state, all the observer objects are automatically notified [5].

The Observer pattern is applied on a very significant feature of our proposed design: the notification mechanism. Notifications are sent when the states of derivatives instruments or portfolios conform to certain predefined rules. For example, when the difference between the market and theoretical price of a derivative becomes large enough to permit arbitrage or when the delta of a portfolio in respect to one its underlying instruments exceeds a certain value. In such cases, users should be notified immediately, in order to take advantage of the arbitrage opportunity or perform portfolio rebalancing.

For simplicity, the NotificationAppService class is defined as observable object and not each Business Object separately. The NotificationAppService class is triggered at constant intervals (e.g. every 60 seconds) by a system timer. It monitors derivatives and portfolios states, sending notifications to observer objects. Observer objects implement the InstrumentListener interface. The EmailAppService and SocketAppService classes, which send notifications via email and TCP/IP respectively, have been defined as observers. Additional observers can be easily introduced. 


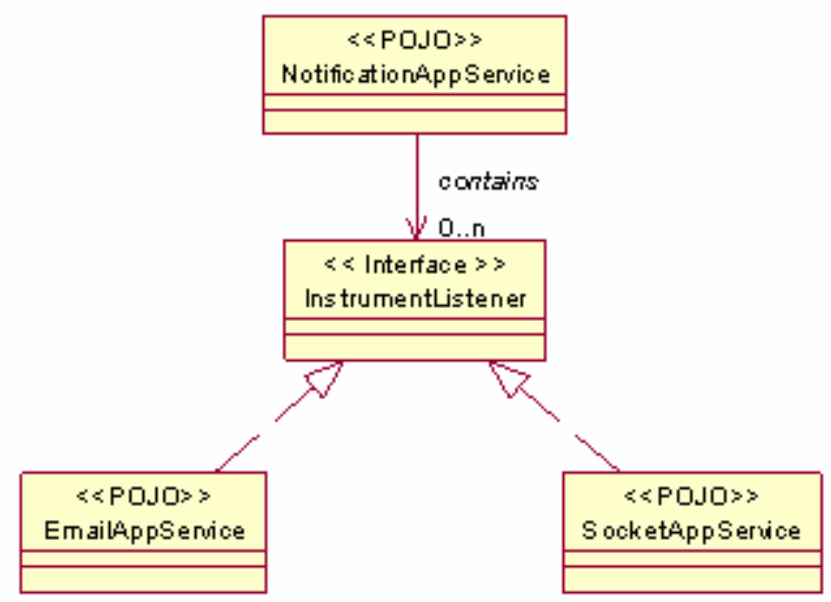

Figure 7: Observer.

\subsection{Integration tier}

\subsubsection{Integration Adapter}

The Adapter pattern converts the interface of an object or system to another interface that a client is capable of using [5]. The Integration Adapter pattern is a special case of the Adapter pattern which refers to the integration with third-party systems that perform similar functionality but provide different interfaces, such as market data feeds. In our design, the IntegrationAdapter interface adopts this pattern. It is implemented by the HTMLAdapter, XMLAdapter and SOAPAdapter classes, which consume market data available in HTML, XML and SOAP format respectively. These classes may be further subclassed to allow data consumption from different providers. This way, additional market data feeds may be introduced with minor modifications.

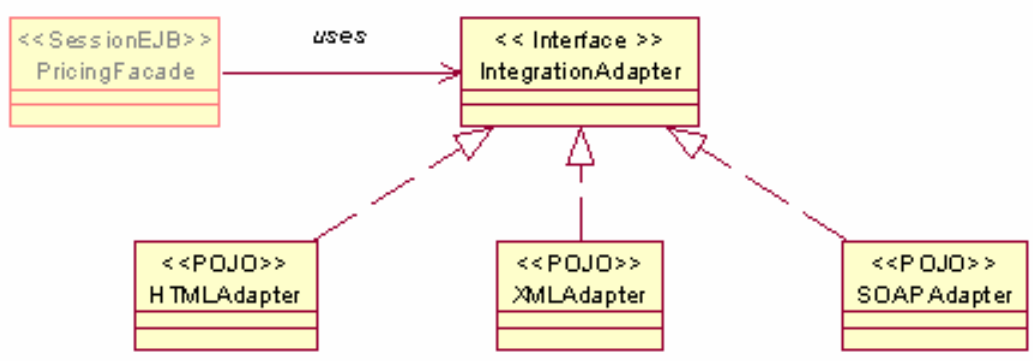

Figure 8: Integration tier design patterns. 


\section{Conclusions}

The present paper aims to combine the theory behind financial derivatives pricing with the latest trends in software engineering, such as the development of web-based applications, the adoption of multi-tiered architectures and the use of design patterns, in order to propose an innovative design of a web-based application for real-time derivatives pricing. Our design is entirely based on the adoption of design patterns, both generic and web-based applications specific, and incorporates some of the principal models for derivatives pricing (Black-Scholes model, binomial methods, Monte Carlo simulation). The introduction of new types of derivatives instruments, additional pricing models and alternative market data feeds is substantially facilitated by our model.

\section{References}

[1] Alur, D., Crupi, J., \& Malks, D., Core J2EE Patterns: Best Practices and Design Strategies, Second Edition, Prentice Hall, 2003.

[2] Birrer, A., \& Eggenschwiler, T., Frameworks in the financial engineering domain: an experience report, Proceedings ECOOP '93, Springer-Verlag: Berlin, LNCS 707, pp. 21-35, 1993.

[3] Duffy, D., Financial Instrument Pricing Using C++, Wiley, 2004.

[4] Eggenschwiler, T., \& Gamma, E., ET++SwapsManager: Using object technology in the financial engineering domain, Proceedings OOPSLA '92, ACM SIGPLAN, 27(10), pp. 166-177, 1992.

[5] Gamma, E., Helm, R., Johnson, R., \& Vlissides, J., Design Patterns: Elements of Reusable Object-Oriented Software, Addison-Wesley, 1995.

[6] Hull, J., Options, Futures and Other Derivatives, Fifth Edition, Prentice Hall, 2003.

[7] Joshi, M., C++ Design Patterns and Derivatives Pricing, Cambridge, 2004.

[8] Koulisianis, M., Tsolis, G., \& Papatheodorou, T., A web-based problem solving environment for solution of option pricing problems and comparison of methods, Proceedings of the International Conference on Computational Science (Part I), pp. 673-682, 2002.

[9] London, J., Modeling Derivatives in C++, Wiley, 2005

[10] Marsura P., A Risk Management Framework for Derivative Instruments, M.Sc. Thesis, University of Illinois, Chicago, 1998.

[11] van der Meij, M., Schouten, D., \& Eliëns, A., Design patterns for derivatives software, ICT Architecture in the BeNeLux, Amsterdam, 1999.

[12] Zhang, J. Q., \& Sternbach, E., Financial software design patterns, Journal of Object-Oriented Programming, 8(9), pp. 6-12, 1996. 